\title{
The Method of Forming the Recognition Signs Based on the Wavelet Transform of Radar Range Portraits
}

Igor V. Sisigin, Konstantin O. Kolesnikov*, Dmitriy A. Ravdin, Artem V. Belyaev and Vladislav V. Maltsev Yaroslavl Higher Military Air Defense College 28 Moskovskiy, Yaroslavl, 150016, Russia

Received 03.12.2016, received in revised form 22.01.2017, accepted 21.02.2017

The article considers the basic of forming high-contrasts features in the problem of radar recognition with wavelet transform.

Keywords: radar recognition, contrast, radar range profiles, wavelet transform, local extremum lines.

Citation: Sisigin I.V., Kolesnikov K.O., Ravdin D.A., Belyaev A.V., Maltsev V.V. The method of forming the recognition signs based on the wavelet transform of radar range portraits, J. Sib. Fed. Univ. Eng. technol., 2017, 10(2), 277-285. DOI: 10.17516/1999-494X-2017-10-2-277-285.

\section{Методика формирования признаков распознавания на основе вейвлет-преобразования \\ радиолокационных дальностных портретов}

\author{
И.В. Сисигин, К.О. Колесников, \\ Д.А. Равдин, А.В. Беляев, В.В. Мальцев \\ Ярославское высшее военное училище противовоздушной обороньы \\ Россия, 150016, Ярославль, Московский пр., 28
}

Рассматривается методика формирования высококонтрастных признаков в задаче радиолокаиионного распознавания с использованием вейвлет-преобразования.

Ключевые слова: радиолокачионное распознавание, контрастность, радиолокационнодальностный портрет, вейвлет-преобразование, линии локальных экстремумов.

(C) Siberian Federal University. All rights reserved

* Corresponding author E-mail address: level1@inbox.ru 


\section{Введение}

Одной из перспективных задач развития радиолокационных систем специального назначения является внедрение новых способов обработки отраженных сигналов с целью классификации объектов локации по их тактическому назначению. В основе решения этой задачи лежат методы радиолокационного распознавания, которые достаточно полно раскрыты в ряде источников [1-7]. В них указано, что наиболее эффективными считаются методы, основанные на анализе признаков в виде радиолокационных дальностных портретов (РЛДП) [5]. Большинство алгоритмов распознавания по РЛДП основаны на байесовском подходе [7]. В этом случае качество принятия решения о принадлежности объекта к заданному классу (типу) оценивается матрицей условных вероятностей распознавания. В $[2,7]$ утверждается, что основным средством повышения качества распознавания служит расширение спектра зондирующих сигналов и увеличение отношения сигнал-шум. Вместе с тем высокая плотность радиолокационных станций в группировке, необходимость решения задач на предельных дальностях, а также возможное радиоэлектронное противодействие ограничивают ширину спектра зондирующих сигналов и достижимое отношение сигнал-шум. В этих условиях качество радиолокационного распознавания с использованием РЛДП возможно повысить только за счет дополнительной обработки, направленной на повышение контрастности признаков.

\section{Контрастность радиолокационных дальностных портретов}

Понятие контрастности РЛДП и ее связь с показателями качества распознавания введены в работе [8]. В ней РЛДП представляется как совокупность нормально распределенных статистически независимых комплексных амплитуд $\eta_{i}$, где $i$ - количество элементов разрешения цели. Вероятность правильного распознавания цели $k$-го класса определяется по формуле

$$
D_{k}=\prod_{l \neq k}^{M}\left[\frac{1}{2}+\frac{1}{2} \Phi\left(\frac{\bar{\rho}_{k l / k}}{\sigma_{k l / k}}\right)\right]
$$

где $\Phi(x)=\frac{2}{\sqrt{2 \pi}} \int_{0}^{x} e^{-\frac{t}{2}} d t-$ интеграл вероятности; $\bar{\rho}_{k l / k}-$ среднее значение коэффициента корреляции $\rho_{k l / l}$ между $k$-м текущим и $l$-эталонным портретом; $\sigma_{k l / k}-$ СКО коэффициента корреляции между $k$-м текущим и $l$-эталонным портретом при наличии цели $k$-го класса.

Из (1) следует, что вероятность правильного распознавания $D_{k}$ зависит от интеграла вероятности $\Phi\left(\frac{\bar{\rho}_{k l / k}}{\sigma_{k l / k}}\right)$. Аргумент интеграла вероятности - это отношение среднего значения коэффициента корреляции к СКО случайной величины $\sigma_{k l / k}$ при наличии цели $k$-го класса, который определяется выражением

$$
\begin{aligned}
& \frac{\bar{\rho}_{k l / k}}{\sigma_{k l / k}}=\frac{\sum_{i=1}^{N}\left[\Delta_{k l}^{i}-\ln \left(1+\Delta_{k l}^{i}\right)\right]}{\sqrt{\sum_{i=1}^{N}\left(\Delta_{k l}^{i}\right)^{2}}} \\
& \Delta_{k l}^{i}=\frac{\sigma_{i^{k}}^{2}+\sigma_{i^{0}}^{2}}{\sigma_{i l}^{2}+\sigma_{i^{0}}^{2}}-1
\end{aligned}
$$


где $\Delta_{k l}^{i}$ - отношение дисперсий коэффициентов корреляции; $\sigma_{i^{0}}^{2}$ - дисперсия собственного шума, одинаковая для всех элементов разрешения.

Величина $\Delta_{k l}^{i}$, характеризующая отклонение от единицы отношений дисперсий $i$-х элементов разрешения $k$-го и $l$-го портретов, может принимать как положительные, так и отрицательные значения. Учитывая, что $\ln (1+x) \approx x-\frac{x^{2}}{2}$, имеем следующие выражения:

$$
\begin{aligned}
& \frac{\bar{\rho}_{k l / k}}{\sigma_{k l / k}}=\frac{1}{2} \sqrt{q_{k l}} ; \\
& q_{k l}=\sum_{i=1}^{N}\left(\Delta_{k l}^{i}\right)^{2} .
\end{aligned}
$$

Величина $q_{k l}$ определена как контрастность между портретом, соответствующим правильному решению о наличии цели $k$-го класса, и другими портретами $l \neq k$. Следовательно, вероятность правильного распознавания $D_{k}$ прямо пропорциональна величине контрастности $q_{k l}$.

Таким образом, при ограничениях на ширину спектра зондирующего сигнала и отношение сигнал-шум качество радиолокационного распознавания можно улучшить только за счет дополнительной обработки РЛДП, обеспечивающей повышение контрастности.

\section{Методика формирования контрастных признаков распознавания}

Ниже представлена методика формирования высококонтрастных признаков распознавания, основанная на линейном разложении исходного РЛДП с помощью двухпараметрических базисных функций.

В основе методики лежит ряд предположений:

1. Контрастность каждого РЛДП определяется относительным объемом информации, отличающей его от других.

2. Совокупность РЛДП в выбранном алфавите классов может быть представлена матрицей $Z=\left\|z_{l, i}\right\|$ размерностью $L \times N$, где $L-$ количество классов в алфавите, $N$ - протяженность (размерность) каждого РЛДП при его представлении в дискретном виде.

3. Показателем контрастности может быть коэффициент парной корреляции Пирсона

$$
\rho_{l, m}=\frac{\sum_{i=1}^{N}\left(\left(Z_{l, i}-\overline{Z_{l, l}}\right)\left(Z_{m, i}-\overline{Z_{m, l}}\right)\right)}{\sqrt{\left(\sum_{i=1}^{N}\left(Z_{l, i}-\overline{Z_{l, l}}\right)^{2} \sum_{i=1}^{N}\left(Z_{m, i}-\overline{Z_{m, l}}\right)^{2}\right)}}
$$

где $-l \neq m$ индексы распознаваемых классов (типов) из выбранного алфавита размерностью L.

4. Интегральный показатель качества системы распознавания - среднее значение условной вероятности ошибки распознавания, зависит от контрастности каждой пары РЛДП:

$$
\overline{P_{\mathrm{ow}}}=\frac{1}{L(L-1)}\left(\sum_{l=1}^{L} \sum_{m=1}^{L} P_{l, m}-\sum_{l=1}^{L} P_{l, l}\right),
$$

где $\left\|P_{l, m}\left(\rho_{l, m}\right)\right\|$ - матрица условных вероятностей распознавания.

5. Линейное разложение РЛДП позволяет выделить его оригинальные составляющие и использовать их для распознавания классов и типов объектов $[9,10]$.

Для линейного разложения сигналов применяются методы, основанные на системах ортогональных функций различного вида. К ним относятся преобразования Фурье, Уолша, 
вейвлет-преобразование, преобразование Карунена-Лоэва (ПКЛ), использующие гармонические функции, функции Радемахера, Уолша, Хаара и др. Результат разложения представляется в виде одно-либо двумерного вектора коэффициентов в зависимости от вида и числа параметров базисных функций.

В контексте решаемой задачи предполагается, что особенности каждого РЛДП локализованы на некотором случайном временном интервале. Учитывая принцип неопределенности Гейзенберга, для выделения характерных особенностей каждого РЛДП методом ортогонального разложения целесообразно использовать базисные функции, локализованные как по времени, так и по частоте (масштабу) $[10,11]$.

Из всех вышеперечисленных преобразований этому требованию удовлетворяет вейвлетпреобразование вида

$$
w_{z}(a, b)=\frac{1}{\sqrt{a}} \int_{-\infty}^{\infty} Z(t) \psi\left(\frac{t-b}{a}\right) d t,
$$

где $Z(t)$ - РЛДП; $\psi_{a, b}(t)$ - базисная вейвлет-функция.

Для дискретных значений параметров $a$ и $b$ результатом вейвлет-преобразования будет матрица $W_{z}=\left\|w_{j, i}\right\|$, где $w_{j, i}=\frac{1}{\sqrt{a_{j}}} \int_{-\infty}^{\infty} Z(t) \psi\left(\frac{t-b_{i}}{a_{j}}\right) d t$.

Механизм вейвлет-преобразования типового РЛДП с использованием ортогонального вейвлет-базиса Хаара приведен на рис. 1. РЛДП задан в виде вектора размерностью N. Для наглядности ось абсцисс масштабирована в единицах длины объекта (с шагом 1 см) и единицах времени (с шагом $\approx 67$ пс).

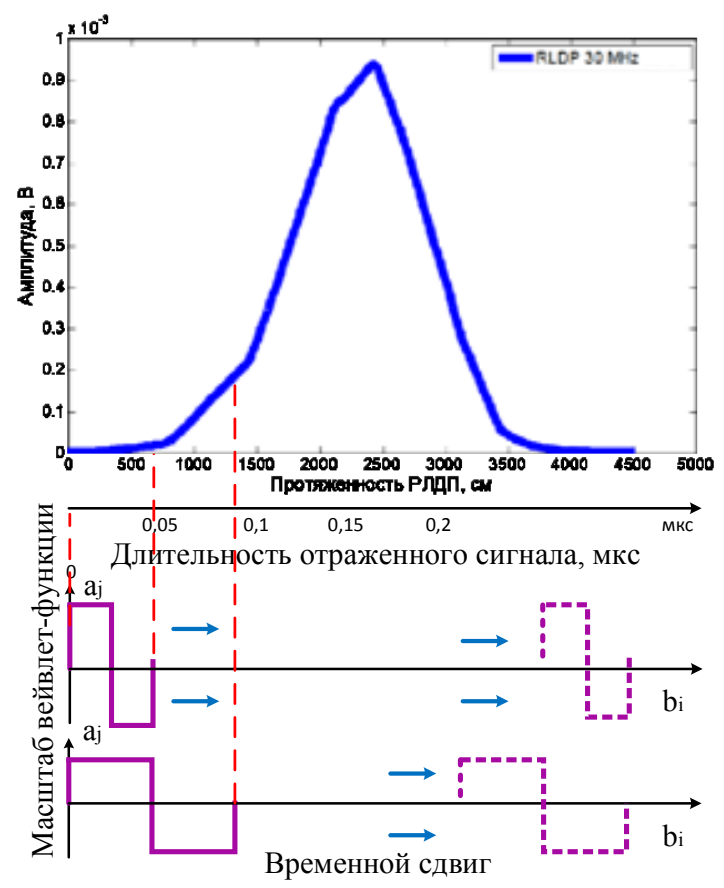

Рис. 1. Механизм вейвлет-преобразования РЛДП с использованием базиса Хаара 
Матрица вейвлет-коэффициентов $W_{z}$ в координатах масштаб - протяженность представлена на рис. 2 в виде поверхности. Каждый вейвлет-коэффициент $w_{j, i}$ характеризует среднюю скорость изменения РЛДП в пределах интервала интегрирования, которая может восприниматься как информационный элемент.

На рис. 2 видно, что поверхность, образуемая вейвлет-коэффициентами $W_{z}$, имеет полимодальный вид. Причем по оси масштаба просматриваются устойчивые локальные экстремумы, характеризующие участок РЛДП с определенной скоростью изменения. Математическое моделирование РЛДП нескольких объектов и вычисление вейвлет-коэффициентов показали наличие экстремумов во всех случаях. Причем их положение было оригинальным для каждого из объектов.

Таким образом, первым этапом методики является разложение исходного РЛДП по ортогональному вейвлет-базису с вычислением матрицы вейвлет-коэффициентов $W_{z}$.

Для выделения локальных экстремумов, характеризующих особенности РЛДП, над матрицей коэффициентов $W_{z}$ проводится операция вычисления первых и вторых разностей с последующим бинарным преобразованием в соответствие с выражением

$$
l w_{j, i}= \begin{cases}1, & \Delta W 1_{j, i}<0 \wedge \Delta W 2_{j, i}<0 \\ 0, & \Delta W 1_{j, i}>0 \vee \Delta W 2_{j, i}>0\end{cases}
$$

где $\Delta W 1_{j, i}=\left(w_{j, i+1}-w_{j, i}\right) \cdot\left(w_{j, i}-w_{j, i-1}\right) ; \Delta W 2_{j, i}=w_{j, i+1}+w_{j, i-1}-2 w_{j, i}$.

На рис. 3 показаны линии локальных экстремумов (ЛЛЭ) матрицы $L w=\left\|l w_{j, i}\right\|$ как результат преобразования (9) матрицы $W_{z}$. Положение и вид ЛЛЭ определяются видом РЛДП. Таким образом, второй этап методики - формирование бинарной матрицы $L w$.

Как видно на рис. 3 и 4, количество ЛЛЭ с увеличением масштаба уменьшается. Это объясняется увеличением длительности базисной функции и интервала интегрирования в (8). В то же время положение ЛЛЭ оказывается более стабильным в пределах близких ракурсов объекта (рис. 4, области, выделенные окружностями). Следовательно, для выделения наиболее устойчивых ЛЛЭ необходимо выполнить параметрическую оптимизацию матрицы $L w$ путем ограничения снизу на некотором заданном уровне.

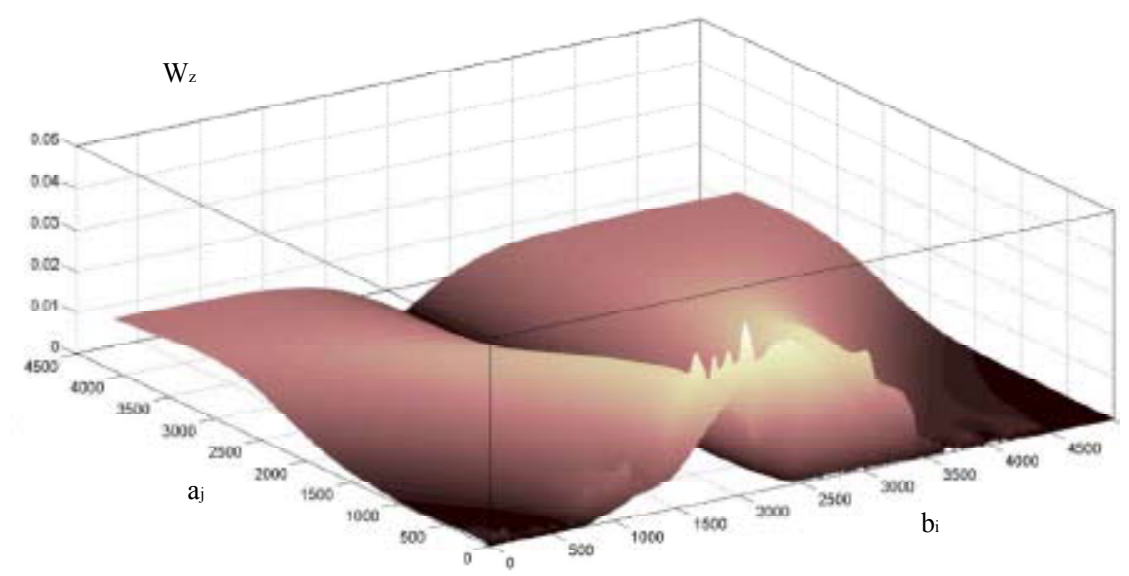

Рис. 2. Поверхность вейвлет-коэффициентов $W_{z}$ 


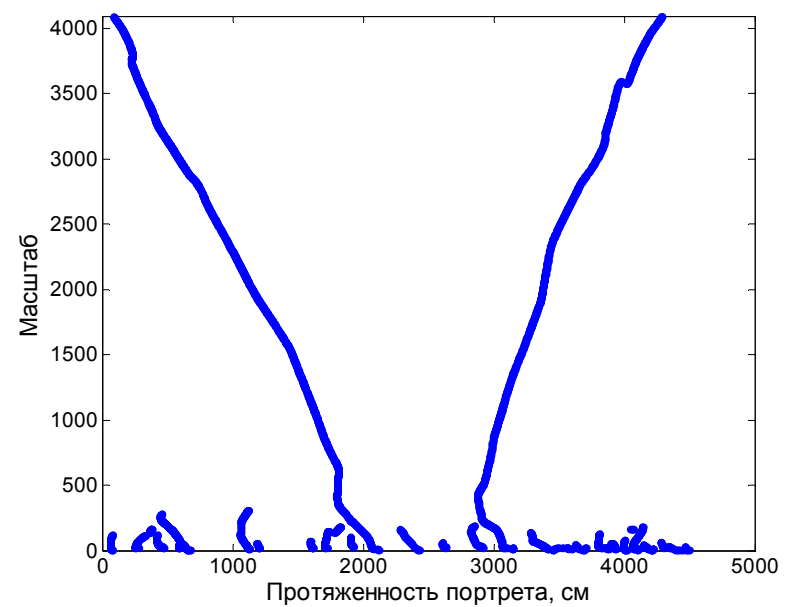

Рис. 3. Линии локальных экстремумов

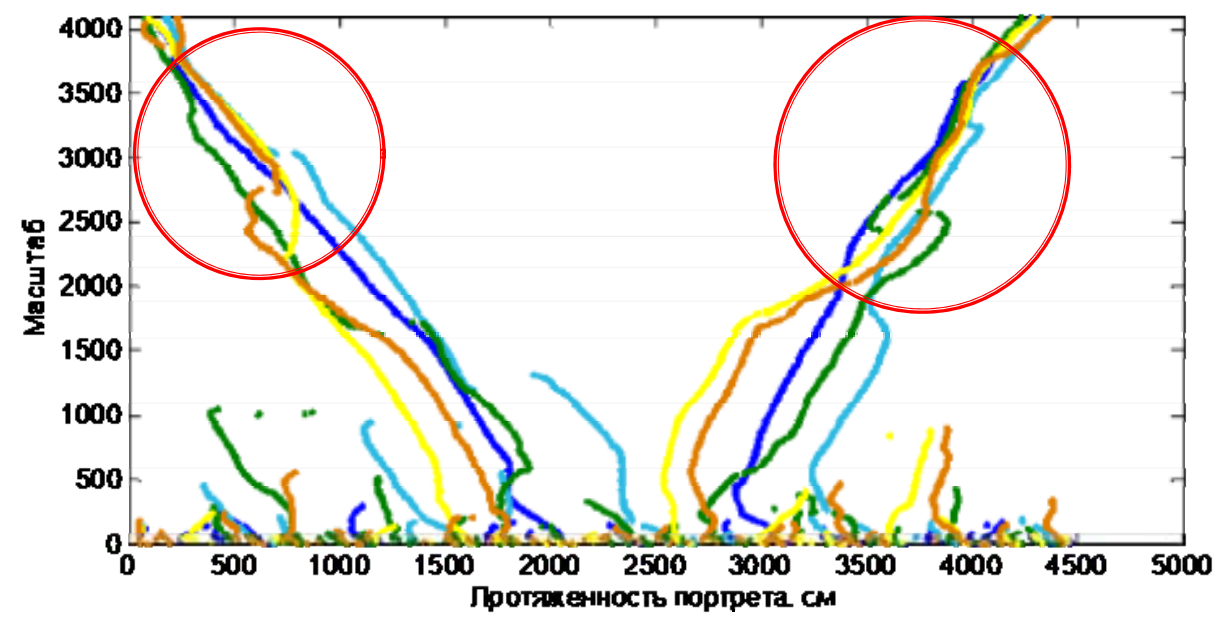

Рис. 4. Семейство из пяти ЛЛЭ для объекта 1 при изменении ракурса с шагом $4^{\circ}$

Мы выбирали уровень ограничения исходя из заданного минимального количества ЛЛЭ для каждого из объектов (РЛДП).

На практике уровень ограничения может быть определен исходя из требуемого значения коэффициента межклассовой корреляции эталонных портретов выбранного алфавита классов (типов).

Таким образом, заключительным этапом методики является операция оптимизации матрицы $L w$ путем выбора уровня ограничения масштаба снизу (рис. 5).

\section{Оценка показателей качества методики формирования признаков распознавания}

Эффективность предложенной методики оценивалась для алфавита типов объектов $(L=4)$ в пределах одного класса, РЛДП и ЛЛЭ которых показаны на рис. 6 и 7 соответственно. 


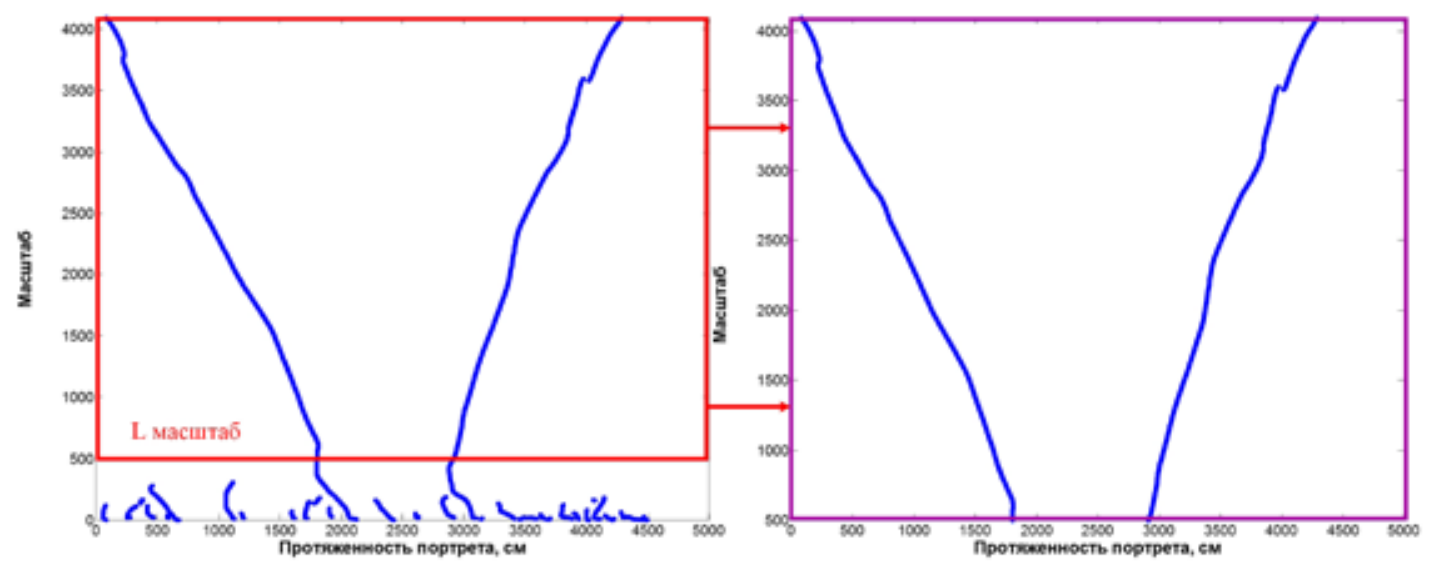

Рис. 5. Линии локальных экстремумов и уровень ограничения масштаба

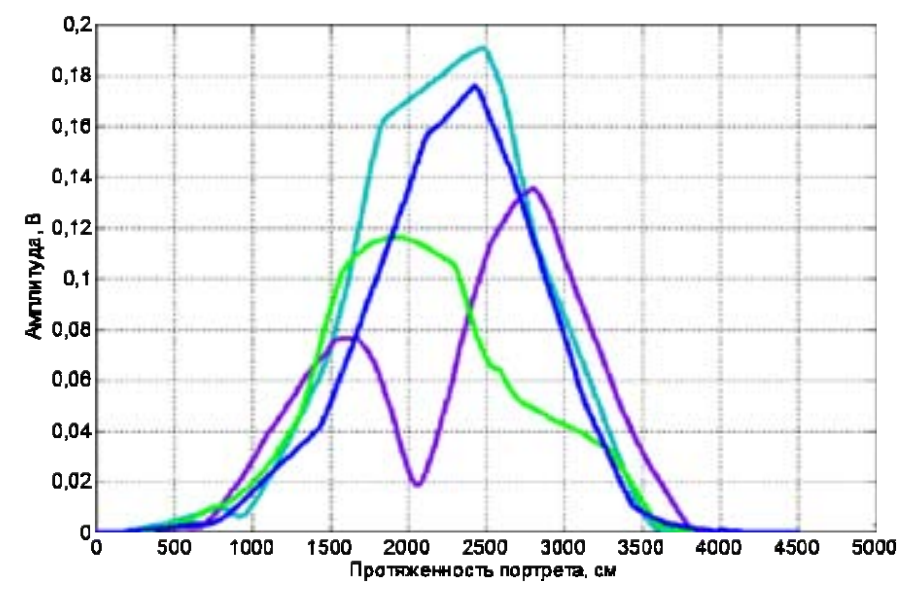

Рис. 6. Семейство РЛДП четырех типов объектов

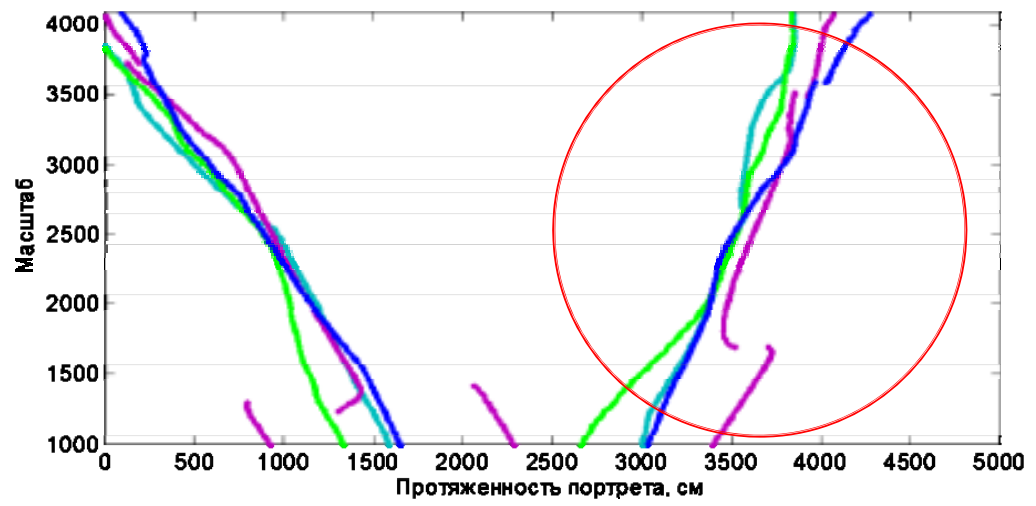

Рис. 7. Семейство ЛЛЭ для объектов различных классов 
Оценка среднего значения коэффициента парной корреляции для всех пар выбранных РЛДП составила $\rho_{l, m}=0,76$, а для ЛЛЭ $\rho_{l, m}=0,03$. Таким образом, контрастность признаков распознавания на основе линий локальных экстремумов вейвлет-коэффициентов по выбранному показателю более чем на порядок превышает контрастность исходных РЛДП.

Результаты математического моделирования устройства распознавания классов (типов) объектов, использующего ЛЛЭ, подтвердили выдвинутую гипотезу о монотонной зависимости показателей качества радиолокационного распознавания от контрастности эталонных признаков.

\section{Выводы}

1. Линейное разложение РЛДП позволяет сформировать устойчивые, компактные, контрастные признаки распознавания.

2. Контрастность признаков распознавания в виде ЛЛЭ зависит от параметров вейвлетпреобразования и выбранных уровней ограничений по оси масштаба.

3. Дальнейшие исследования будут проводиться с большим алфавитом классов и пониженным отношением сигнал-шум.

\section{Список литературы}

[1] Небабин В.Г., Сергеев В.В. Методы и техника радиолокаиионного распознавания. М.: Радио и связь, 1984. 152 с. [Nebabin VG, Sergeev VV Methods and technology of radar recognition. Moscow, Radio and Communication, 1984. 152 p. (in Russian)].

[2] Кузьмин С.З. Цифровая радиолокачия. Введение в теорию. Киев: Изд-во КВіЦ, 2000. 428 c. [Kuzmin S.Z. Digital radar. Introduction to the theory. Kiev, KViTS, 2000. 428 p. (in Russian)].

[3] Горелик А.Л. и др. Современное состояние проблемы распознавания: Некоторые аспекты. М.: Радио и связь, 1985. 160 с. [Gorelik A.L. And others. Current state of the problem of recognition: Some aspects. Moscow, Radio and Communication, 1985. 160 p. (in Russian)].

[4] Слюсарь Н.М. Вторичная модуляция радиолокачионных сигналов динамическими объектами. Смоленск: ВА ВПВО ВС РФ, 2006. 173 с. [Slyusar N.M. Secondary modulation of radar signals by dynamic objects. Smolensk, WA WPVO RF Armed Forces, 2006. 173 p. (in Russian)].

[5] Лещенко С.П. Развитие теории и техники радиолокационного распознавания воздушных целей. Прикладная радиоэлектроника, 2009, 4(8), 490-496. [Leschenko S.P. Development of radar theory and technology Recognition of air targets. Applied radioelectronics, 2009, 4(8), 490-496 (in Russian)].

[6] Охрименко А.Е. Основы радиолокации и радиоэлектронная борьба. Ч. 1. М: Воениздат, 1983. 475 с. [Okhrimenko A.E. Fundamentals of radar and electronic warfare. Part 1. Moscow, Military Publishing, 1983. 475 p. (in Russian)].

[7] Ширман Я.Д. и др. Радиолокационное распознавание и методы математического моделирования. Научно-технические серии. М.: ИПРЖР, 2000. 96 с. [Shirman Ya.D. Radar recognition and methods of mathematical modeling. Scientific and technical series. Moscow, IPRZHR, 2000. 96 p. (in Russian)].

[8] Дворников С.В., Сауков А.М. Метод распознавания радиосигналов на основе вейвлетпакетов. Научное приборостроение, 2004, 14(1), 85-93 [Dvornikov S.V., Saukov A.M. The method 
of recognition of radio signals based on wavelet packets. Scientific instrument making, 2004, 14(1), 85-93 (in Russian)].

[9] Козинов И.А. Обнаружение локальных свойств анализируемых сигналов и процессов с использованием вейвлет-преобразования. Информационно-управляющие системы, 2015, 1 (74), 21-28 [I.Kozinov. Detection of local properties of analyzed signals and processes using wavelet transform. Information-control systems, 2015, 1 (74), 21-28 (in Russian)].

[10] Добеши И. Десять лекиий по вейвлетам. Ижевск: НИЦ «Регулярная и хаотическая динамика», 2001. 464 с. [Dobesi I. Ten lectures on wavelets. Izhevsk: SRC «Regular and chaotic dynamics», 2001. 464 p. (in Russian)]. 